\title{
Was ist "Aktivierung" in der Arbeitsmarktpolitik?
}

Kai Marquardsen

Der Begriff „Aktivierung“ erfreut sich gegenwärtig großer Popularität. Bemerkenswert ist aber, dass bislang nicht konsistent definiert ist, was sich hinter dem Begriff verbirgt. Ziel des Beitrags ist es, die Bedeutung(en) von Aktivierung in der deutschen Arbeitsmarktpolitik aufzuzeigen. Dafür werden die Verhaltensanforderungen an die Individuen analysiert, die der Aktivierungspolitik implizit sind.

\section{Einleitung}

Obwohl viele ihrer Instrumente nicht gänzlich neu sind, markiert die aktivierende Arbeitsmarktpolitik eine grundlegende Neuausrichtung der deutschen Arbeitsmarktund Sozialpolitik. Sie besteht im Kern in einer Fokussierung auf das individuelle Verhalten der Arbeitslosen (Bartelheimer 2005, S. 55f.; Dingeldey 2005, S. 6). Arbeitslosigkeit wird als Folge individueller Verhaltensdefizite interpretiert. Entsprechend ist es das Ziel der Arbeitsmarktpolitik, durch „verhaltensstützende und -beeinflussende Maßnahmen" (Hartz et al. 2002) eine Verhaltensänderung herbeizuführen und „erwünschtes"Verhalten herzustellen (Dingeldey 2005, S. 22f.). Die Verantwortung für das persönliche Arbeitsmarktschicksal (und damit letztlich für die gesellschaftliche Misere der Arbeitslosigkeit) wird auf diese Weise individualisiert, womit die Arbeitsmarktpolitik entlastet wird (Brütt 2003, S. 650).

Vor dem Hintergrund, dass der erhoffte Effekt der aktivierenden Arbeitsmarktpolitik bislang weitgehend ausgeblieben ist (Bundesregierung 2006), sind Zweifel an den Grundprämissen der Aktivierungspolitik angebracht. So wird unterstellt, dass das Problem der Arbeitslosigkeit vor allem auf die mangelnde Motivation und Bereitschaft der Arbeitslosen zurückzuführen sei. Dabei wird ausgeblendet, dass eine wichtige Ursache auch in der fehlenden gesamtwirtschaftlichen Nachfrage nach Arbeitskräften liegt und somit struktureller Natur ist (Trube 2003, S. 302). Dennoch gilt es, das Aktivierungsparadigma ernst zu nehmen. Denn für die Betroffenen ist es gerade aufgrund seiner fragwürdigen Grundprämissen folgenreich.

Ziel dieses Beitrags ist es, die Frage „Was ist Aktivierung?“" durch den Blick auf die Anforderungen zu beantworten, die an das Verhalten der Individuen gestellt werden. Dies erfordert, alle potenziellen Wirkungsdimensionen der Aktivierungspolitik gleichermaßen zu berücksichtigen. Selbst wenn einiges darauf hindeutet, dass die repressive Variante in der Praxis dominiert (Lessenich 2005; Brütt 2003; Spindler 2003; Trube 2003), lässt sich Aktivierung nicht darauf reduzieren. Eine umfassende Wirkungsanalyse kann letztlich nur auf der Grundlage empirischer Untersuchungen erfolgen. Dies wiederum setzt voraus, dass das Aktivierungsparadigma zunächst in seiner ganzen konzeptionellen Breite erfasst wird. Um die unterschiedlichen Facetten der Aktivierungspolitik transparent zu machen, werden im folgenden Abschnitt drei Zieldimensionen der aktivierenden Arbeitsmarktpolitik identifiziert. AnschlieBend wird aufgezeigt, welche arbeitsmarktpolitischen Maßnahmen in welcher Dimension wirksam sind.

\section{Dimensionen der Aktivierung}

Der Begriff „Aktivierung“ suggeriert, dass sich die betroffenen Arbeitslosen in einem Zustand der Passivität befinden, aus dem es sie wachzurütteln gilt. Da Arbeitslosigkeit als Verhaltensdefizit der Erwerbslosen gedeutet wird, wird das individuelle Verhalten zum Gegenstand der arbeitsmarktpolitischen Intervention. Anhand der einschlägigen Literatur lassen sich drei zentrale Zieldimensionen der aktivierenden Arbeitsmarktpolitik identifizieren: die Bereitschaft der Arbeitslosen zur Annahme einer Beschäftigung (Verfügbarkeit), die aktive Verwertung der eigenen Arbeitskraft (Eigenverantwortung) und die (Wieder-)Herstellung bzw. der Erhalt von Arbeitsmarktnähe (Beschäftigungsfähigkeit).

\subsection{VERFÜGBARKEIT}

Ein erheblicher Teil der Auseinandersetzung mit der Aktivierungspolitik dreht sich um den Aspekt einer erweiterten Verfügbarkeit für den Arbeitsmarkt - insbesondere dann, wenn es um die Zwangsaspekte der neuen Arbeitsmarktgesetzgebung geht (Völker 2005; Brütt 2003; Spindler 2003). Allerdings ist Verfügbarkeit nicht ausschließlich durch repressive Maßnahmen zu erzielen. Verfügbarkeit meint Beschäftigungsbereitschaft. Dies kann einerseits bedeuten, dass Verfügbarkeit über Instrumente hergestellt werden soll, die mit negativen Sanktionen im Falle mangelnder Bereitschaft verbunden sind (Variante Fordern). Andererseits lassen sich aber auch Instrumente identifizieren, die eine Verfügbarkeit für den Arbeitsmarkt ermöglichen sollen, wenn die Bereitschaft zur Aufnahme einer Beschäftigung zwar vorhanden, die Verfügbarkeit aber durch besondere Hemmnisse eingeschränkt ist (Variante Fördern) (Abschnitt 3.1).

\subsection{EIGENVERANTWORTUNG}

Die zweite Zieldimension der aktivierenden Arbeitsmarktpolitik ist die der Eigenverantwortung (Bothfeld et al. 2005; Dahme/Wohlfahrt 2005; Lessenich 2005). Auch in dieser Dimension sind zwei Varianten zu unterscheiden. Das Ziel einer aktiveren Rolle des Individuums bei der Vermarktung seiner eigenen Arbeitskraft wird also auf zwei Wegen zu erreichen versucht: zum einen mittels eines erhöhten Drucks auf die

Kai Marquardsen, M. A., promoviert zum Thema "Wechselwirkungen zwischen Aktivierung und sozialen Netzwerken " an der Universität Jena. Arbeitsschwerpunkte: Arbeits- und Industriesoziologie, Netzwerkforschung.

e-mail: kai.marquardsen@uni-jena.de 
Arbeitslosen und der Sanktionierung mangelnder Eigenverantwortung (Variante Fordern); zum anderen durch die Ermöglichung eigenverantwortlichen Verhaltens, indem vorhandene Eigenaktivitäten unterstützt oder Barrieren beseitigt werden, die der Übernahme von Eigenverantwortung entgegenstehen (Variante Fördern) (Abschnitt 3.2).

\subsection{BESCHÄFTIGUNGSFÄHIGKEIT}

Die dritte Zieldimension ist schließlich die Beschäftigungsfähigkeit. Hierbei geht es um die (Wieder-)Herstellung bzw. um den Erhalt von Arbeitsmarktnähe. Beschäftigungsfähigkeit beschreibt „die Fähigkeit einer Person, auf der Grundlage ihrer fachlichen und Handlungskompetenzen, Wertschöpfungs- und Leistungsfähigkeit ihre Arbeitskraft anbieten zu können und damit in das Erwerbsleben eintreten zu können, ihre Arbeitsstelle zu halten oder, wenn nötig, sich eine neue Erwerbsbeschäftigung zu suchen" (Blancke et al. 2000, S. 9). Die Instrumente in dieser Dimension zielen auf die Herstellung von Arbeitsmarktnähe bzw. die Verhinderung von Arbeitsmarktferne. Eben daran lässt sich der Unterschied zwischen Fördern und Fordern verdeutlichen: Während es in der fördernden Variante um einen gezielten Ausgleich von Qualifikationsdefiziten geht, zielt die fordernde Variante lediglich darauf, der fortschreitenden „Entwöhnung" von der Erwerbsarbeit entgegenzuwirken (Abschnitt 3.3).

\section{Instrumente der Aktivierung}

\subsection{INSTRUMENTE IN DER DIMENSION "VERFÜGBARKEIT “}

Zunächst werden jene Instrumente betrachtet, die mit negativen Sanktionen im Falle mangelnder Verfügbarkeit verbunden sind (Variante Fordern). Im Anschluss daran werden die Instrumente behandelt, die Beschäftigungsbereitschaft durch den Abbau von Verfügbarkeitshemmnissen ermöglichen sollen (Variante Fördern).

Variante Fordern

(1) Zumutbarkeit: Als Instrument des Forderns von Verfügbarkeit wirken die ver- schärften Zumutbarkeitsregeln. Dies gilt insbesondere im SGB II-Bereich, wo die Regelungen des Bundessozialhilfegesetzes übernommen wurden. Berufs-, Qualifikations- und Tarifschutzbestimmungen waren hier bereits 1998 außer Kraft gesetzt worden. Aber auch im Bereich des SGB III gelten mit zunehmender Dauer der Arbeitslosigkeit verschärfte Definitionen, was zumutbar ist. Dies gilt sowohl bei der Höhe des Arbeitsentgelts als auch für einen Wohnortwechsel. Wichtig ist in diesem $\mathrm{Zu}$ sammenhang die Umkehr der Beweislast: Nicht mehr der Träger der Grundversorgung muss die Zumutbarkeit einer Beschäftigung rechtfertigen, sondern der oder die Arbeitslose hat die Unzumutbarkeit einer Beschäftigung nachzuweisen. Verbindlich werden diese Regelungen durch Sanktionen in Form von Sperrzeiten, die Absenkung der Regelleistungen oder das vollständige Erlöschen des Anspruchs. Im Bereich des SGB II gilt mit dem Fortentwicklungsgesetz seit August 2006 (Deutscher Bundestag 2006), dass bei der ersten Ablehnung eines Vermittlungsangebots zunächst die Unterkunftszahlungen gekürzt werden. Bei der zweiten Ablehnung erfolgt eine Kürzung des Regelsatzes und der Mietzahlungen um $60 \%$. Bei der dritten Ablehnung werden die Leistungen komplett gestrichen. Der Zeitraum, innerhalb dessen die Pflichtverletzung verfällt, wurde von drei auf zwölf Monate ausgedehnt. ${ }^{1}$

Sanktionen sind auch bei der Verletzung der frühzeitigen Meldepflicht vorgesehen: Personen, deren versicherungspflichtiges Arbeitsverhältnis endet, müssen sich unverzüglich nach Kenntnis des Beendigungszeitpunkts persönlich bei der Agentur für Arbeit arbeitsuchend melden. Für befristet Beschäftigte gilt eine Meldepflicht drei Monate vor Ablauf des Arbeitsvertrags. Bei Versäumnis kommt es zu einer Minderung des Anspruches von bis zu $50 €$ für jeden Tag der verspäteten Meldung. Laut Fortentwicklungsgesetz sollen schließlich Arbeitslose, die erstmalig das Arbeitslosengeld II beziehen, ein Sofortangebot erhalten, dessen Ablehnung sanktioniert wird. Ziel dieses Instruments ist es, die Verfügbarkeit für den Arbeitsmarkt vor dem Eintritt in den Leistungskreis des SGB II zu prüfen und damit Arbeitslosigkeit und Entgeltersatzleistungen zu vermeiden (Bundesregierung 2006, S. 119).

(2) Kontrolle: Zusätzlich gibt es zahlreiche Instrumente, die zur Kontrolle der Motiva- tion und Beschäftigungsbereitschaft eingesetzt werden. Dazu gehören a) die Beauftragung von Trägern mit Eingliederungsmaßnahmen, b) die Personal-ServiceAgenturen (PSA), c) die Beauftragung Dritter mit der Vermittlung, d) Vermittlungsgutscheine, e) Eignungsfeststellungsund Trainingsmaßnahmen sowie f) Arbeitsgelegenheiten.

ad a) Im Falle der Beauftragung von Trägern mit Eingliederungsmaßnahmen handelt es sich um eine Ermessensleistung der Agentur. Die Vergütung der Träger besteht aus einer Fallpauschale und einer Erfolgsprämie für jede erfolgreiche Vermittlung, die zeitlich gestaffelt ausbezahlt wird. Bei jeder fehlgeschlagenen Vermittlung verlangen die Agenturen einen festen Betrag von den Maßnahmeträgern zurück. Über die Tatsache hinaus, dass dieses Instrument genutzt wird, um die Verfügbarkeit am Arbeitsmarkt zu kontrollieren, dürfte sein aktivierender Effekt auch durch das Eigeninteresse des Maßnahmenträgers am Vermittlungserfolg verstärkt werden. Voraussetzung für die Wirksamkeit des Instruments ist jedoch die sanktionsbehaftete Zuweisung von Arbeitslosen in solche Maßnahmen, da nur auf diese Weise ein Fordern von Seiten der Agentur möglich ist.

ad b) Dies gilt auch mit Blick auf die PSA. Diese stellen Arbeitslose befristet ein und verleihen sie an andere Betriebe. Die Zuweisung der Arbeitslosen zu einer PSA erfolgt durch die Agenturen. Dies führt in der Praxis zu Konflikten mit den Maßnahmeträgern, die wegen des erfolgsabhängigen Honorars ein Interesse an leicht vermittelbaren Arbeitslosen haben.

ad c) Bei der Beauftragung von Dritten mit der Vermittlung wird die Auswahl der TeilnehmerInnen ebenfalls von den Agenturen getroffen. Im Gegensatz zur Beauftragung von Trägern und zu den PSA stellt dieses Instrument eine Pflichtleistung dar, auf die Arbeitslose nach sechsmonatiger Arbeitslosigkeit einen Rechtsanspruch haben. Die Agenturen können hierbei Dritte mit der gesamten Vermittlung, mit Teilaufgaben der Vermittlung oder mit der Stellenakquisition beauftragen. Allerdings

\footnotetext{
Für Jugendliche unter 25 Jahren führt die Ablehnung eines Vermittlungsangebots oder der $A b$ bruch einer Maßnahme zu einem sofortigen und vollständigen Wegfall der Leistungen. Ihnen wird in diesem Fall nur die Übernahme der Kosten für Unterkunft und Heizung gewährt.
} 
wird auch dieses Instrument häufig zur Überprüfung der Beschäftigungsbereitschaft angewendet. Für die Arbeitslosen hat eine Ablehnung der Maßnahme leistungsrechtliche Konsequenzen.

ad d) Als doppeldeutig ist die Wirkung der Vermittlungsgutscheine einzuschätzen: Sie können im Sinne des Förderns wirken, wenn sie für die eigenverantwortliche Arbeitssuche genutzt werden. Dies setzt jedoch ein hohes Maß an Eigeninitiative voraus (Abschnitt 3.2). In der Praxis werden die Vermittlungsgutscheine stattdessen oftmals benutzt, um die Verfügbarkeit von Arbeitslosen zu prüfen.

ad e) Ähnlich doppeldeutig sind die Eignungsfeststellungs- und Trainingsmaßnahmen: Wenn sie zur Prüfung der Beschäftigungsbereitschaft eingesetzt werden, wirken sie als Instrument des Forderns. Sie können jedoch auch zur Behebung geringerer Qualifikationsdefizite dienen (Abschnitt 3.3).

ad f) Auch Arbeitsgelegenheiten sind dann zu den fordernden Instrumenten der Aktivierungspolitik zu zählen, wenn sie angeboten werden, um die Verfügbarkeit der Arbeitslosen zu kontrollieren. Die Arbeitsgelegenheiten kommen ausschließlich im SGB II-Bereich zur Anwendung. Es wird zwischen der Entgelt- und der Mehraufwandsvariante unterschieden. Erstere ist eine sozialversicherungspflichtige Beschäftigung, bei der das übliche Arbeitsentgelt anstatt des Arbeitslosengeldes II ausgezahlt wird. Bei der Mehraufwandsvariante („1Euro-Job") handelt es sich um eine gemeinnützige Beschäftigung, bei der die Arbeitslosen neben dem Arbeitslosengeld II eine Mehraufwandsentschädigung von $1-2 €$ pro Stunde erhalten. Insbesondere dieses Mittel verdeutlicht, dass die aktivierende Arbeitsmarktpolitik zur Ausweitung prekärer und atypischer Beschäftigung beitragen kann (Brinkmann et al. 2006, S. 78f.; Lessenich 2005, S. 25f.; Walther 2003, S. 302), da ihre Instrumente bestehende sozialpolitische Standards unterlaufen. Dies verweist nicht zuletzt auf die erzieherische Komponente der Aktivierungspolitik: Die Betroffenen lernen, Arbeit sprichwörtlich „um jeden Preis“ zu akzeptieren.

(3) Individualisierung: Mit Umsetzung der Arbeitsmarktreformen wurde die Erstellung von Bewerberprofilen als Standardvorgang bei der Arbeitslosigkeitsmeldung eingeführt. Ziel des Profilings ist es, die für die Vermittlung erforderlichen beruflichen und persönlichen Merkmale von Arbeitslosen festzustellen. Auf dieser Grundlage erfolgt eine Prognose der Arbeitsmarktchancen, die zur Unterscheidung zwischen „Kundengruppen“ führt. Der Ressourceneinsatz variiert entlang der Gruppen. Im Falle der „Marktkunden“ wird davon ausgegangen, dass eine Integration in den Arbeitsmarkt auch ohne intensivere Unterstützung gelingt. Im Falle der „Beratungskunden" wird ein Unterstützungsbedarf angenommen, wobei erwartet wird, dass die Aufnahme einer Erwerbsarbeit vor Eintritt in den Förderkreis des SGB II erfolgen kann. ${ }^{2}$ Bei den „Betreuungskunden“ dagegen werden kaum Arbeitsmarktchancen erwartet, weshalb diese in der Praxis nur ein geringes $\mathrm{Ma}$ an Unterstützung erhalten (Bundesregierung 2006, S. IX-X).

Die Bedeutung der Bewerberprofile ist zum einen darin zu sehen, dass sie die Voraussetzung für einen zielgerichteten Instrumenteneinsatz sind. Darüber hinaus ist dem Profiling aber auch eine unmittelbar aktivierende Wirkung eigen: Es beinhaltet einen systematischen Abgleich zwischen den vorhandenen und den gewünschten Eigenschaften der Person. Bildlich gesprochen wird den Arbeitslosen durch das Profiling ein Spiegel vorgehalten, der ihren Marktwert sichtbar macht. Insofern hat das Instrument eine sekundäre Wirkung: Es weist soziale Rollen zu.

Bei den Eingliederungsvereinbarungen handelt es sich um einen Vertragsabschluss zwischen Arbeitsagentur und dem oder der Arbeitslosen, auch wenn die Arbeitslosen de facto nicht den Status eines vollwertigen Marktteilnehmers haben (Brütt 2003, S. 648). Hiermit werden sowohl die Vermittlungsbemühungen der Agentur als auch die Eigenbemühungen der Arbeitslosen verbindlich festgeschrieben.

(4) Finanzieller und sozialer Druck: Als sanktionsbehaftetes Fordern von Verfügbarkeit wirkt im Bereich des SGB II auch die Möglichkeit, Lebensmittelgutscheine anstelle von Bargeld an Arbeitslose auszugeben. ${ }^{3}$ Da mit den Gutscheinen nur ,lebensnotwendige Güter" erworben werden können, ist mit diesem Instrument eine erhebliche Einschränkung der Lebensqualität verbunden. Damit steigt nicht nur der unmittelbare Druck zur Annahme einer Beschäftigung. Vielmehr werden die individuellen Erwartungen und Gewohnheiten unmittelbar beeinflusst. Darüber hinaus haben die Lebensmittelgutscheine eine so- zial stigmatisierende Wirkung: Der Status der Person als Leistungsempfänger wird beim Einkauf öffentlich, was mittelbar den Zwang zur Verhaltensänderung verstärken kann.

Auf eine Erhöhung des finanziellen und sozialen Drucks zur Aufnahme einer Beschäftigung wirkt im Rechtskreis des SGB II zudem die Bemessung der Leistungsansprüche am Einkommen der Bedarfsgemeinschaft hin, wonach das Einkommen des Lebenspartners oder der Lebenspartnerin in die Berechnung der Leistungsansprüche einbezogen wird. Mit dem Fortentwicklungsgesetz gilt auch in Bezug auf die Bedarfsgemeinschaften eine Beweislastumkehr: Wer mit anderen in einem Haushalt zusammenlebt, muss selbst nachweisen, dass es sich nicht um eine eheähnliche Gemeinschaft handelt.

Als Maßnahmen der Verfügbarkeitsprüfung sind schließlich die Ausweitung der Kontrollen bei Verdachtsfällen von Leistungsmissbrauch (z. B. Hausbesuche und Telefonabfragen) sowie die erweiterten $\mathrm{Zu}$ griffsmöglichkeiten auf die Daten anderer Behörden (z. B. Finanzbehörden, Meldebehörden, Kraftfahrtbundesamt) zu deuten. Zum einen kann wiederum der drohende Entzug von Leistungen die Arbeitsbereitschaft erhöhen. Zum anderen kann schon die Möglichkeit, jederzeit in die Privatsphäre der Arbeitslosen einzudringen, eine „aktivierende“"Wirkung haben.

\section{Variante Fördern}

$\mathrm{Zu}$ den fördernden arbeitsmarktpolitischen Instrumenten in der Dimension Verfügbarkeit gehören unter anderem der Eingliederungszuschuss, der Einstellungszuschuss bei Neugründung und bei Vertretung, der Beitragsbonus und die Förderung der Teilhabe Behinderter. Diese Instrumente ermöglichen die Verfügbarkeit für den Arbeitsmarkt, wenn diese durch Hemmnisse eingeschränkt ist. So sind z. B. die Zielgruppen des Eingliederungszuschusses Ältere und Langzeitarbeitslose sowie Personen mit anderen „Vermittlungshemmnis-

\footnotetext{
Je nach Einschätzung der Motivation und Qualifikation wird zwischen „Beratungskunden aktivieren" und "Beratungskunden fördern" unterschieden.

3 Diese Praxis war bislang nur bei Asylsuchenden üblich. Zu den Parallelen zwischen Arbeitsmarktreformen und Migrationsmanagement in Deutschland vgl. Kopp (2006).
} 
sen“, wie schlechten Deutschkenntnissen oder Drogenabhängigkeit (Bundesregierung 2006, S. 172). In allen genannten Fällen handelt es sich allerdings um Lohnzuschüsse an den Arbeitgeber, um ihm einen Anreiz zur Beschäftigung zu bieten. Demgegenüber ist die Entgeltsicherung für Ältere ein Zuschuss zum Arbeitsentgelt und zum Beitrag zur Rentenversicherung an über 50-Jährige, wenn diese die Arbeitslosigkeit beenden oder vermeiden. Als Instrument des Förderns von Verfügbarkeit wirkt ferner die Unterstützung der Beratung und Vermittlung. Hierunter fällt z. B. die Kostenerstattung bei Bewerbungen. Ähnlich ermöglichen Mobilitätshilfen eine Verfügbarkeit für den Arbeitsmarkt, indem sie Fahrt-, Lebenshaltungs- und Umzugskosten ersetzen.

\subsection{INSTRUMENTE IN DER DIMENSION „EIGENVERANT- WORTUNG"}

Die Instrumente in der Dimension Eigenverantwortung zielen auf eine aktive Rolle des Individuums bei der Vermarktung der eigenen Arbeitskraft. Sie beinhalten einerseits Maßnahmen zur Sanktionierung fehlender Eigenverantwortung und -initiative (Variante Fordern). Andererseits sollen sie eigenverantwortliches Verhalten ermöglichen, indem vorhandene Eigenaktivitäten unterstützt oder Barrieren beseitigt werden, die der Übernahme von Eigenverantwortung entgegenstehen (Variante Fördern).

\section{Variante Fordern}

$\mathrm{Zu}$ den Instrumenten des Forderns von Eigenverantwortung gehören verschärfte $Z u$ mutbarkeitsregeln, Sperrzeiten, die Absenkung der Regelleistungen bzw. das Erlöschen des Anspruchs auf Lohnersatzleistungen, die Umkehr der Beweislast sowie die frühzeitige Meldepflicht. Über den Aspekt des Einforderns von Verfügbarkeit (Abschnitt 3.1) hinaus wirken diese Instrumente darauf hin, Arbeitslose - unter Androhung von Sanktionen - zu eigenverantwortlichen Marktsubjekten zu „erziehen“. Eigenverantwortung ist hier also eindeutig negativ ausgelegt: Sind die Betroffenen nicht bereit, das jeweilige Angebot anzunehmen, müssen sie die leistungsrechtlichen Konsequenzen tragen.

Auch die Eingliederungsvereinbarungen wirken als forderndes Instrument in der
Dimension Eigenverantwortung. Der Vertragsabschluss symbolisiert die Eigenverantwortung des Arbeitslosen für die Integration in den Arbeitsmarkt. Die Verantwortung für das Schicksal des Einzelnen auf dem Arbeitsmarkt wird zunehmend individualisiert (Abschnitt 4).

Schließlich sind die Beauftragung von Trägern mit Eingliederungsmaßnahmen, die Beauftragung Dritter mit der Vermittlung sowie die Personal-Service-Agenturen als Instrumente des Einforderns von Eigenverantwortung zu verstehen. Bei nicht konformem Verhalten - z. B. im Falle einer Ablehnung oder eines Abbruchs solcher Maßnahmen - können Sanktionen erfolgen.

\section{Variante Fördern}

Zu den Instrumenten der Förderung selbstständiger Tätigkeiten zählten bislang die auf den Bereich des SGB III beschränkten Instrumente des Überbrückungsgelds und des Existenzgründungszuschusses („Ich$\left.A G^{\prime \prime}\right)$. Sie wurden inzwischen durch den Gründungszuschuss ersetzt. Jenseits der Frage nach dem Erfolg dieser Instrumente kann die Förderung von Selbstständigkeit für das Leitbild der Aktivierung durchaus als paradigmatisch verstanden werden, da mit ihr ein Höchstmaß an Eigenverantwortung und -initiative verbunden ist. Dies gilt insbesondere im Falle der Alleinselbstständigkeit. Bislang ungenutzte individuelle Ressourcen sollen auf diese Weise mobilisiert und verwertet werden.

Das Einstiegsgeld im Bereich SGB II kann sowohl bei Aufnahme einer selbstständigen Tätigkeit (Variante Entgelt) als auch einer sozialversicherungspflichtigen Beschäftigung (Variante Beschäftigung) eingesetzt werden. Der Schwerpunkt der Förderung liegt jedoch eindeutig auf der Variante Entgelt (BA 2006, S. 12f.). Ihre Wirksamkeit entspricht jener der oben genannten Instrumente im SGB III. Aber auch die Variante Beschäftigung kann über das Mittel finanzieller Anreize als Instrument des Förderns von Eigenverantwortung wirksam werden.

Die aktivierende Wirkung des Bildungsgutscheins liegt darin, dass er es der zu fördernden Person überantwortet, einen geeigneten Weiterbildungsträger $\mathrm{zu}$ suchen. Allerdings werden aufgrund der vorgeschriebenen Verbleibsquote von $70 \%$ vor allem solche Arbeitslosen gefördert, die lediglich ein geringes Qualifizierungsdefizit haben und voraussichtlich schnell wieder eine Beschäftigung finden. Insofern ist der Bildungsgutschein nur bedingt ein Instrument der Aktivierung, da er eine vorhandene Motivation und Arbeitsmarktnähe voraussetzt. Dies gilt auch für den Vermittlungsgutschein, der vor allem von der Gruppe der „Marktkunden“ aktiv genutzt wird (Bundesregierung 2006, S. 122f.), während er bei den „Betreuungskunden“ überwiegend zur Prüfung der Verfügbarkeit eingesetzt wird. Daran wird deutlich, dass einzelne Instrumente der aktivierenden Arbeitsmarktpolitik zur Reproduktion ungleicher Arbeitsmarktchancen und -risiken beitragen.

\subsection{INSTRUMENTE IN DER DIMENSION "BESCHÄFTIGUNGSFÄHIGKEIT“}

Die Instrumente in der Dimension Beschäftigungsfähigkeit zielen auf die (Wieder-)Herstellung bzw. den Erhalt von Arbeitsmarktnähe. Für die Zuordnung zu den Varianten Fordern oder Fördern ist einerseits entscheidend, ob die Maßnahmenteilnahme erzwungen wird oder freiwillig erfolgt. Andererseits gilt es zu berücksichtigen, ob die Befähigung der betreffenden Person im Vordergrund steht oder lediglich das Ziel, einer fortschreitenden „Entwöhnung" von Erwerbsarbeit entgegenzuwirken ist. Zur Variante Fördern werden hier nur solche Maßnahmen gezählt, bei denen es gezielt darum geht, die Beschäftigungsfähigkeit mittels der gezielten Förderung von Fähigkeiten und Kenntnissen zu verbessern.

\section{Variante Fordern}

Bei den Arbeitsbeschaffungsmaßnahmen $(A B M)$ handelt es sich um eine durch pauschalisierte Lohnkostenzuschüsse geförderte Beschäftigung, die im öffentlichen Interesse sein soll und deren Ergebnis nicht überwiegend erwerbswirtschaftlichen Zwecken dienen darf. ABM zählen zu den „klassischen“ Instrumenten der Arbeitsmarktpolitik. Trotzdem kann ihre Wirkung in einem allgemeinen Sinne als „aktivierend“ bezeichnet werden: Sie verhindern, dass die Betroffenen in die „Passivität“ abgleiten. Dies als „Fördern“ zu bezeichnen, ist jedoch unangebracht, da es hier nicht um die Ermöglichung von Beschäftigungsfähigkeit, sondern um die Verhinderung von Arbeitsmarktdistanz geht. Eine ergänzende oder zusätzliche Qualifi- 
kation findet in der Regel nicht statt. Obwohl also die Teilnahme an einer Maßnahme von Seiten der Arbeitslosen erwünscht sein kann, sind ABM zu den Instrumenten des Forderns von Beschäftigungsfähigkeit zu zählen. Gleiches gilt auch für das Instrument der Arbeitsgelegenheiten (Abschnitt 3.1).

Die Personal-Service-Agenturen (PSA) wiederum sollen die Arbeitslosen in verleihfreien Zeiten bei der Suche nach einer regulären Beschäftigung sowie bei der Qualifizierung und Weiterbildung unterstützen. Allerdings ist das Instrument bislang nur mäßig erfolgreich (Bundesregierung 2006, S. 139ff.). Insofern fordern PSA über die sanktionsbehaftete Maßnahmenzuweisung zwar die (Wieder-)Herstellung von Beschäftigungsfähigkeit ein, bieten aber umgekehrt nur wenig langfristige Integrationsperspektiven.

\section{Variante Fördern}

Das zentrale Instrument des Förderns von Beschäftigungsfähigkeit ist die berufliche Aus- und Weiterbildung. Hier wird durch Qualifizierung langfristig Beschäftigungsfähigkeit ermöglicht. Dies gilt auch für den Bildungsgutschein. Schließlich können auch Eignungsfeststellungs- und Trainingsmaßnahmen als Instrumente des Förderns von Beschäftigungsfähigkeit wirken, wenn sie nicht zur Überprüfung der Verfügbarkeit (Abschnitt 3.1), sondern zur Behebung von Qualifizierungsdefiziten eingesetzt werden. In der Praxis erweist es sich jedoch als Problem, dass die Chancen, in den Genuss einer solchen Förderung zu kommen, ungleich verteilt sind: Es werden vor allem diejenigen gefördert, bei denen eine „Investition" in eine Qualifizierung kurzfristig lohnenswert erscheint (Dahme/Wohlfahrt 2005, S. 17).

\section{Individualisierung und Privatisierung}

Quer zu den drei vorgenannten Dimensionen liegt die Dimension einer verstärkten Individualisierung der Verantwortung für das eigene Arbeitsmarktschicksal. Diese vollzieht sich z. B. aufgrund der Tatsache, dass die Leistungen der Grundversorgung durch die Politik des Forderns unter Vorbehalt gestellt werden: Lehnen Arbeitslose ein Jobangebot ab, müssen sie die Konsequenzen in Form verschärfter Sanktionen tragen, während zugleich die Beweislast für die Unzumutbarkeit des Angebots bei ihnen liegt. Andererseits wird die Individualisierung, z. B. auch durch die Förderung selbstständiger Tätigkeiten, vorangetrieben, da das erfolgreiche Bestehen als „Unternehmer der eigenen Arbeitskraft" eine aktive Selbstvermarktung erfordert. Die Tendenz zur Individualisierung drückt sich schließlich auch darin aus, dass es den Arbeitslosen zunehmend selbst zufällt, ihre eigene Beschäftigungsfähigkeit erfolgreich (wieder-)herzustellen.

Mit Blick auf andere Instrumente stellt sich die Individualisierung jedoch als widersprüchlich dar. So wirkt die Zuordnung der Arbeitslosen zu einer von vier „Kundengruppen" im Sinne einer Kategorisierung des Individuums. Zugleich ist dieser Vorgang ein in hohem Maße standardisiertes Verfahren, das der betroffenen Person kaum gerecht werden kann. Widersprüchlich ist die Individualisierungstendenz auch mit Blick auf die Bemessung der Leistungsansprüche am Einkommen der Bedarfsgemeinschaft. Hier wird die Verantwortung für Erfolg und Versagen am Arbeitsmarkt an die Angehörigen der Arbeitslosen weitergegeben. Diese werden gewissermaßen in "Sippenhaft“ genommen. Insofern erscheint es sinnvoll, nicht von einer Individualisierung, sondern von einer Privatisierung der Verantwortung durch die aktivierende Arbeitsmarktpolitik zu sprechen: Die Bewältigung von Arbeitsmarktchancen und -risiken fällt verstärkt dem Einzelnen und seinem sozialen Umfeld zu. Private Beziehungen und ehemals separate Räume der privaten Lebenswelt werden zum Gegenstand arbeitsmarktstrategischen Denkens und Handelns. Eine solche Privatisierung der Verantwortung für das individuelle Arbeitsmarktschicksal muss von den Betroffenen nicht zwingend als Gefährdung wahrgenommen werden. Einige mögen sie durchaus als Chance begreifen. Dennoch dürfte die faktische Konzentration der Arbeitsmarktpolitik auf die Instrumente des Forderns zur Folge haben, dass für viele Arbeitslose insbesondere die Privatisierung von Arbeitsmarktrisiken zur dominanten Erfahrung wird.

\section{Fazit: Was ist Aktivierung?}

Durch die aktivierende Arbeitsmarktpolitik wird das individuelle Verhalten der Arbeitslosen zum Bezugspunkt der arbeitsmarktpolitischen Intervention. Aktivierung bedeutet, durch geeignete Verhaltensanreize unerwünschtes Verhalten $\mathrm{zu}$ verhindern und erwünschtes Verhalten herzustellen. Es wurden drei Dimensionen herausgearbeitet, auf die sich der Versuch einer solchen Verhaltenssteuerung bezieht. Jedes Instrument der Aktivierungspolitik lässt sich mindestens einer dieser drei Wirkungsdimensionen zuordnen, wobei es möglich ist, dass ein Instrument zugleich in mehreren Dimensionen funktionell ist. Zusätzlich lässt sich jede der drei Dimensionen von Aktivierung intern in eine negative, erzwungene Variante (Fordern) und eine positive, ermöglichende Variante (Fördern) differenzieren, wodurch insgesamt sechs mögliche Ausprägungen unterschieden werden können (Tabelle 1).

Die Forderung nach Verfügbarkeit, Eigenverantwortung und Beschäftigungsfähigkeit wirkt über die negative Sanktionierung unerwünschten Verhaltens. Im Zuge der Arbeitsmarktreformen wurden bekannte Sanktionen (z. B. Sperrzeiten, Leistungskürzungen) ausdifferenziert und verschärft und durch Sanktionen ergänzt, die auf eine mittelbare Wirkung über das soziale Umfeld der Person ausgerichtet sind (Lebensmittelgutscheine, Bedarfsgemeinschaften). Die Förderung von Verfügbarkeit, Eigenverantwortung und Beschäftigungsfähigkeit soll dagegen erwünschtes Verhalten durch positive Verhaltensanreize ermöglichen.

Der aktivierenden Arbeitsmarktpolitik ist ein erzieherischer Aspekt inhärent: Die Arbeitslosen sollen zu nützlichen Marktsubjekten geformt werden. Dieses Ziel ist allen Instrumenten der Aktivierungspolitik gemeinsam. Allerdings hängt die Möglichkeit einer erfolgreichen Aktivierung maßgeblich davon ab, wie nah oder fern die erwünschten Eigenschaften den Arbeitslosen sind: Eine Förderung kommt in der Praxis überwiegend solchen Arbeitslosen zugute, bei denen ein zeitnaher Integrationserfolg erwartet wird, während Personen mit einem hohen Betreuungsbedarf kaum unterstützt werden (Bundesregierung 2006, S. X). Die Chancen, in den Genuss einer 
Förderung zu kommen, sind also ungleich verteilt. In diesem Ungleichgewicht zeigt sich ein Widerspruch zwischen Leitbild und Realität der aktivierenden Arbeitsmarktpolitik: Ausgerechnet diejenigen, die besonders "förderungswürdig" sind, werden oftmals von der Förderung ausgeschlossen.

Dies mag einerseits finanztechnische bzw. haushaltspolitische Gründe haben. Ein weiterer Grund liegt sicherlich in der Verwaltungspraxis, d.h. im Umgang mit den gesetzlichen Vorgaben auf der Ausführungsebene. Letztlich ist diese Schieflage jedoch schon auf der Ebene der Gesetzgebung und in den Verordnungen angelegt. Dies zeigt sich nicht nur im Fortentwicklungsgesetz der Großen Koalition, durch das eine noch stärkere Verschiebung vom Fördern zum Fordern stattgefunden hat. Vielmehr sind es die dem Leitbild der Aktivierung zugrunde liegenden Grundprämissen selbst, die eine Betonung der „Pflichten“ der Arbeitslosen befördern. Eine sinnvolle Politik der Aktivierung müsste stattdessen das bedarfsorientierte Fördern und die „Befähigung“ (Rabe/Schmid 2000) der Betroffenen stärker in den Vordergrund stellen, da dies erst die Voraussetzungen für eigenverantwortliches Handeln schafft. Als in vielerlei Hinsicht positiv lässt sich hier auf das dänische Modell (Goul Andersen 2003; van Oorschot/Abrahamson 2003; van Berkel et al. 2002) verweisen. Entscheidend ist, dass den Forderungen auch Wahlmöglichkeiten gegenüberstehen müssen (Walther 2003, S. 304) und dass die Arbeitslosen Aktivierung nicht nur als Bedrohung, sondern als Chancenerweiterung wahrnehmen können (Lodemel/Trickey 2001, S. 134). ${ }^{3}$

Was also ist Aktivierung? Ziel des Beitrags war es, sich der Antwort durch eine Analyse der Anforderungen zu nähern, welche die aktivierende Arbeitsmarktpolitik an die arbeitslosen Individuen stellt. Ein abschließendes Urteil erfordert weiterführende Untersuchungen: zum einen des Verhältnisses zwischen Fordern und För-

\begin{tabular}{|c|c|c|}
\hline \multirow[t]{2}{*}{ Dimension } & \multicolumn{2}{|l|}{ Variante } \\
\hline & Fordern & Fördern \\
\hline Verfügbarkeit & $\begin{array}{l}\text { Zumutbarkeitsregeln } \\
\text { Sperrzeiten } \\
\text { Absenkung/Erlöschen des } \\
\text { Leistungsanspruchs } \\
\text { Umkehr der Beweislast } \\
\text { Frühzeitige Meldepflicht } \\
\text { Eignungsfeststellung/Training } \\
\text { Beauftragung von Trägern mit Eingliederung } \\
\text { Beauftragung von Dritten mit Vermittlung } \\
\text { Vermittlungsgutscheine } \\
\text { Profiling } \\
\text { Eingliederungsvereinbarungen } \\
\text { Arbeitsgelegenheiten } \\
\text { PSA } \\
\text { Lebensmittelgutscheine } \\
\text { Bedarfsgemeinschaften }\end{array}$ & $\begin{array}{l}\text { Eingliederungszuschuss } \\
\text { Einstellungszuschüsse } \\
\text { Beitragsbonus } \\
\text { Förderung der Teilhabe Behinderter } \\
\text { Entgeltsicherung für Ältere } \\
\text { Unterstützung bei Beratung und } \\
\text { Vermittlung } \\
\text { Mobilitätshilfen }\end{array}$ \\
\hline $\begin{array}{l}\text { Eigenverant- } \\
\text { wortung }\end{array}$ & $\begin{array}{l}\text { Zumutbarkeitsregeln } \\
\text { Sperrzeiten } \\
\text { Absenkung/Erlöschen des } \\
\text { Leistungsanspruchs } \\
\text { Umkehr der Beweislast } \\
\text { Frühzeitige Meldepflicht } \\
\text { Eingliederungsvereinbarungen } \\
\text { Beauftragung von Trägern } \\
\text { Beauftragung Dritter } \\
\text { PSA }\end{array}$ & $\begin{array}{l}\text { Überbrückungsgeld } \\
\text { Existenzgründungszuschuss } \\
\text { Einstiegsgeld } \\
\text { Bildungsgutschein } \\
\text { Vermittlungsgutschein } \\
\text { Beauftragung Dritter }\end{array}$ \\
\hline $\begin{array}{l}\text { Beschäftigungs- } \\
\text { fähigkeit }\end{array}$ & $\begin{array}{l}\text { ABM } \\
\text { Arbeitsgelegenheiten } \\
\text { PSA }\end{array}$ & $\begin{array}{l}\text { Berufl. Aus- und Weiterbildung } \\
\text { Bildungsgutschein } \\
\text { Eignungsfeststellung/Training }\end{array}$ \\
\hline
\end{tabular}

dern in der arbeitsmarktpolitischen Praxis. Hier gilt es, nicht nur die Relevanz der verschiedenen Instrumente anhand der realen Fallzahlen und der eingesetzten Finanzmittel zu betrachten, sondern auch die Förderstrukturen. Des Weiteren sollte die Frage nach dem Verhältnis zwischen dem Leitbild der aktivierenden Arbeitsmarktpolitik, seiner Umsetzung in Verordnungen und seiner praktischen Anwendung vertieft werden. Hier könnte sich zeigen, dass von Aktivierung als einer konsistenten Strategie nicht die Rede sein kann. Stattdessen ist denkbar, dass "Aktivierung“ auf den verschiedenen Ebenen unterschiedliche und möglicherweise sogar widersprüchliche Bedeutungen hat. Entscheidend ist jedoch, dass sie als Anforderung an das Verhalten der Individuen dennoch überaus wirksam ist. Ein erheblicher Forschungsbedarf besteht deshalb vor allem bezüglich der individuellen und gesellschaftlichen Folgen der Aktivierungspolitik: Wie gehen die Betroffenen mit den Erfahrungen der Aktivierung um? Und wie wirken sich die Anforderungen an die Verfügbarkeit, die Eigenverantwortung und die Beschäftigungsfähigkeit der Individuen in sozialer Hinsicht aus? Die hier entwickelte Analyseperspektive kann für solche vertiefenden Untersuchungen als Grundlage dienen.

3 Ein grundsätzliches Problem ist jedoch die ausschließliche Orientierung der Aktivierungspolitik an der Erwerbsarbeit als zentralem gesellschaftlichem Integrationsmodus: Anstatt die Individuen „um jeden Preis“ in den Arbeitsmarkt integrieren zu wollen, sollte die Etablierung alternativer Formen der sozialen Anerkennung und Zugehörigkeit zugelassen und befördert werden. Als Voraussetzung dafür bedürfte es aber einer bedingungslosen (und mehr als nur existenzsichernden) Grundsicherung. 
Bundesagentur für Arbeit (BA) (2006): Monatsbericht Januar, http://www.pub.arbeitsamt.de/hst/services/statistik/000100/html/ monat/200604.pdf

Bartelheimer, P. (2005): Moderne Dienstleistungen und Erwerbsfürsorge. Fallbearbeitung nach SGB II als Gegenstand soziologischer Forschung, in: SOFI-Mitteilungen 33, S. 55-79

Blancke, S. /Roth, C./Schmid, J. (2000): Employability („Beschäftigungsfähigkeit") als Herausforderung für den Arbeitsmarkt - Auf dem Weg zur flexiblen Erwerbsarbeitsgesellschaft. Eine Konzept- und Literaturstudie, Arbeitsbericht Nr. 157 der Akademie für Technikfolgeabschätzung, Stuttgart

Bothfeld, S. /Gronbach, S./Seibel, K. (2005): Eigenverantwortung in der Arbeitsmarktpolitik: zwischen Handlungsautonomie und Zwangsmaßnahmen, WSI-Diskussionspapier Nr. 134, http://www.boeckler.de/pdf/ p_wsi_diskp_134.pdf

Brinkmann, U./Dörre, K./Röbenack, S./Kraemer, K./Speidel, F. (2006): Prekäre Arbeit. Ursachen, Ausmaß, soziale Folgen und subjektive Verarbeitungsformen unsicherer Beschäftigungsverhältnisse. Gutachten für die Friedrich-Ebert-Stiftung

Brütt, C. (2003): Von Hartz zur Agenda 2010. Die Realpolitik im „aktivierenden Sozialstaat" ", in: Prokla 133, S. 645-665

Bundesregierung (2006): Die Wirksamkeit moderner Dienstleistungen am Arbeitsmarkt. Bericht 2005 der Bundesregierung zur Wirkung der Umsetzung der Vorschläge der Kommission Moderne Dienstleistungen am Arbeitsmarkt (ohne Grundsicherung für Arbeitssuchende),

http://doku.iab.de/externe/2006/k060201a02.pdf

Dahme, H.-J./Wohlfahrt, N. (2005): Sozialinvestitionen. Zur Selektivität der neuen Sozialpolitik und den Folgen für die Soziale Arbeit, in: Dies. (Hrsg.): Aktivierende Soziale Arbeit. Theorie - Handlungsfelder - Praxis, Baltmannsweiler, S. 6-20

Deutscher Bundestag (2006): Entwurf eines Gesetzes zur Fortentwicklung der Grundsicherung für Arbeitssuchende. BT-Drucksache 16/1410 vom 09.05., http://dip.bundestag.de/btd/16/014/1601410.pdf Dingeldey, I. (2005): Welfare State Transformation between "Workfare“ and an „Enabling“ State. A comparative Analysis, TranState Working Papers No. 21, Bremen 2005, http://www.staatlichkeit.uni-bremen.de/ homepages/dingeldey/download.php?ID=23\&SPRACHE=DE\&TABLE= AP\&TYPE=PDF

Goul Andersen, Jørgen (2003): Citizenship Politics: Activation, Welfare and Employment in Denmark. Paper prepared for the conference Social Governance in the Global Era - Beyond 20th Century's Social Democracy. Hokkaido University, Oct. 14-17, Sapporo, Japan
Hartz, P. et al. (2002): Moderne Dienstleistungen am Arbeitsmarkt - Vorschläge der Kommission zum Abbau der Arbeitslosigkeit und zur Umstrukturierung der Bundesanstalt für Arbeit, Berlin

Kopp, H. (2006): Ausbeutung ohne Grenzen. Hartz IV, Migrationsmanagement und Kämpfe dagegen, in: Agenturschluss (Hrsg.): Schwarzbuch Hartz IV. Sozialer Angriff und Widerstand - eine Zwischenbilanz, Hamburg/Berlin, S. 89-97

Lessenich, S. (2005): "Activation without work". Das neue Dilemma des konservativen Wohlfahrtsstaates, in: Dahme, H.-J./Wohlfahrt, N. (Hrsg.): Aktivierende Soziale Arbeit. Theorie - Handlungsfelder - Praxis, Baltmannsweiler, S. 21-29

Lodemel, I./Trickey, H. (2001): Ein neuer Vertrag für Sozialhilfe, in: Stelzer-Orthofer, C. (Hrsg.): Zwischen Welfare und Workfare. Soziale Leistungen in der Diskussion, S. 123-165

Rabe, B./Schmid, G. (2000): Strategie der Befähigung: Zur Weiterentwicklung der Arbeitsmarkt- und Rentenpolitik, in: WSI-Mitteilungen 5 , S. 305-313

Spindler, H. (2003): Fordern und Fördern - Auswirkungen einer sozialpolitischen Strategie auf Bürgerrechte, Autonomie und Menschenwürde, in: Sozialer Fortschritt. Unabhängige Zeitschrift für Sozialpolitik XI/XII, S. 296-301

Trube, A. (2003): Überfordern und Hinausbefördern - Prämissen, Praxis und Probleme aktivierender Sozial- und Arbeitsmarktpolitik, in: Sozialer Fortschritt. Unabhängige Zeitschrift für Sozialpolitik XI/XII, S. 301-305 Trube, A. (2004): Die neue deutsche Arbeitsmarktpolitik und der Wandel des Sozialstaats, in: Sozialer Fortschritt. Unabhängige Zeitschrift für Sozialpolitik III, S. 62-68

Van Berkel, R./Hornemann Møller, I. (Hrsg.) (2002): Active Social Policies in the EU. Inclusion Through Participation?, Bristol

Van Oorschot, W./Abrahamson, P. (2003): The Dutch and Danish Miracles Revisited: A Critical Discussion of Activation Policies in Two Small Welfare States, in: Social Policy \& Administration 37, pp. 288-304 Völker, W. (2005): Aktivierende Arbeitsmarktpolitik. Auf dem Weg zu mehr Zwang und Existenzdruck, in: Dahme, H.-J./Wohlfahrt, N. (Hrsg.): Aktivierende Soziale Arbeit. Theorie - Handlungsfelder - Praxis, Baltmannsweiler, S. 70-87

Walther, A. (2003): Aktivierung: Varianten zwischen Erpressung und Empowerment. Für eine Erweiterung des Diskurses zum aktivierenden Staat im internationalen Vergleich, in: Neue Praxis. Zeitschrift für Sozialarbeit, Sozialpädagogik und Sozialpolitik 3/4, S. 288-305 